BMJ Open

Diabetes

Research

\& Care

\title{
Pharmacovigilance assessment of the association between Fournier's gangrene and other severe genital adverse events with SGLT-2 inhibitors
}

\author{
Gian Paolo Fadini (10 ,' Mayur Sarangdhar, ${ }^{2}$ Fabrizio De Ponti, ${ }^{3}$ Angelo Avogaro, ${ }^{1}$ \\ Emanuel Raschi (i) ${ }^{3}$
}

To cite: Fadini GP

Sarangdhar M, De Ponti F, et al. Pharmacovigilance assessment of the association between Fournier's gangrene and other severe genital adverse events with SGLT-2 inhibitors. BMJ Open Diab Res Care 2019;7:e000725. doi:10.1136/ bmjdrc-2019-000725

- Additional material is published online only. To view please visit the journal online (http://dx.doi.org/10.1136/ bmjdrc-2019-000725).

Received 6 July 2019 Revised 14 August 2019 Accepted 12 September 2019

Check for updates

C Author(s) (or their employer(s)) 2019. Re-use permitted under CC BY-NC. No commercial re-use. See rights and permissions. Published by BMJ.

${ }^{1}$ Department of Medicine, University of Padova, Padova, Italy

${ }^{2}$ Cincinnati Children's Hospital Medical Center, Cincinnati, Ohio, USA

${ }^{3}$ Department of Medical and Surgical Sciences, University of Bologna, Bologna, Italy

Correspondence to Professor Gian Paolo Fadini; gianpaolofadini@hotmail.com

\section{ABSTRACT}

Objective Sodium glucose cotransporter-2 inhibitors (SGLT2i) exert cardiorenal protection in people with diabetes. By inducing glycosuria, SGLT2i predispose to genital infections. In addition, rare occurrence of Fournier's gangrene $(\mathrm{FG})$ has been reported. We aimed to investigate such association through the U.S. Food and Drug Administration (FDA) adverse event (AE) reporting system (FAERS).

Research design and methods We mined the FAERS up to 2018q3 (before FDA warning about SGLT2i-associated $\mathrm{FG}$ ) to retrieve reports including $\mathrm{FG}$ as an AE and SGLT2i as suspect or concomitant drugs, and calculated proportional reporting ratios (PRR).

Results We retrieved 47 cases of $F G$ and 17 cases of other severe AEs of the genital area associated with SGLT2i. Patients with FG were $\sim 10$ years older than those with other severe genital AEs. Overall, $77 \%$ occurred in men. Three patients were concomitantly treated with systemic immunosuppressive drugs. Increased reporting frequency emerged for SGLT2i compared with other drugs, with a PRR ranging from 5 to 10 . The disproportional reporting of FG with SGLT2i remained robust and consistently significant when restricting to the period when SGLT2i were available, to reports filed for glucose-lowering medications or for drugs with the diabetes indication, and after refining the definition of $F G$. FG was disproportionally associated with psoriasis and with the combination of immunosuppressants and SGLT2i.

Conclusions Although causality cannot be demonstrated, SGLT2i may predispose to $F G$ and other severe genital AEs. Since the use of SGLT2i is expected to increase significantly, clinicians should be aware of these severe, although rare, AEs and their predisposing factors.

\section{INTRODUCTION}

Sodium glucose cotransporter-2 inhibitors (SGLT2i) are glucose-lowering medications (GLMs) provided with glycaemic and extraglycaemic effects. ${ }^{1}$ By inhibiting glucose and sodium resorption from the renal tubule, SGLT2i promote glycosuria and natriuresis, thereby reducing glycaemia, body weight and blood pressure. Cardiovascular outcome trials have demonstrated reduced risk of major

\section{Significance of this study}

What is already known about this subject?

- Sodium glucose cotransporter-2 inhibitors (SGLT2i) can protect from adverse cardiovascular and rena outcomes of diabetes.

- However, severe, although very rare, adverse events have been reported during therapy with SGLT2i, including Fournier's gangrene (FG).

What are the new findings?

- In the U.S. Food and Drug Administration adverse event reporting system, we retrieved 47 cases of FG and 17 cases of other severe adverse events of the genital area reported with SGLT2i.

- Increased reporting frequency of such events emerged for SGLT2i compared with other antidiabetic drugs, with disproportionality measures consistently ranging from 5 to 10 .

- FG was disproportionally associated with psoriasis and with the combination of immunosuppressants and SGLT2i.

How might these results change the focus of research or clinical practice?

- Since the use of SGLT2i is expected to increase significantly due to favourable cardiorenal effects, clinicians should be aware that they can rarely lead to severe adverse events of the genital area, including $\mathrm{FG}$.

adverse cardiovascular events and a consistent reduction in the incidence of hospitalisation for heart failure (HHF) in patients with type 2 diabetes (T2D) and cardiovascular disease (CVD) treated with SGLT2i. ${ }^{2}$ The reduction in HHF risk was also observed in patients with T2D without a history of CVD or prior HHF episodes, ${ }^{3}$ and solid data indicate that SGLT2i prevent future adverse renal outcomes of T2D. ${ }^{24}$ Based on these data, SGLT2i are being used by a progressively increasing number of patients worldwide. 
The most common adverse events (AEs) during therapy with SGLT2i are genital infections, ${ }^{5}$ which are mild and resolve completely with or without treatment in most cases. Dehydration, hypovolemia and acute kidney injury are less common AEs. ${ }^{6}$ In addition, SGLT2i have been associated with an increased occurrence of rare but severe AEs, including bone fractures, ${ }^{7}$ diabetic ketoacidosis (DKA) ${ }^{8}$ and below-knee amputations. ${ }^{9}$ The risk for DKA is well documented and related to the drug's mechanism of action, ${ }^{10}$ whereas the increased incidence of fractures and amputations has been reported inconsistently, ${ }^{11}$ and have no clear mechanistic explanation.

In August 2018, the U.S. Food and Drug Administration (FDA) issued a warning that SGLT2i may cause a severe form of perineal necrotising fasciitis (NF) known as Fournier's gangrene (FG). This was based on the identification of 12 cases of FG occurred in patients taking SGLT2i and reported to the FDA from March 2013 to May 2018 or found in the medical literature. ${ }^{12}{ }^{13}$ For comparison, the FDA safety announcement noted that only six cases of FG were identified in review of other GLM classes over a period of $>30$ years. ${ }^{14}$ A complete report up to January 2019 found a total of 55 cases of FG in patients taking SGLT2i and 19 in patients on other GLMs. ${ }^{15}$ To date, no formal quantification of the risk of FG associated with SGLT2i is available. We herein provide a comprehensive analysis of suspected FG reported in association with SGLT2i to the FDA AE reporting system (FAERS), along with other severe AEs of the genital area. For the first time, we performed a disproportionality analysis (DA) to size the dimension of this new SGLT2iassociated AE.

\section{RESEARCH DESIGN AND METHODS}

\section{Data sources and study design}

The public FAERS database from 2004q1 to 2018q3 contains 11525276 reports of AEs and medication errors voluntarily submitted by healthcare professionals, consumers and drug companies from all over the world (serious reports from Europe and non-US countries are also collected). The report form contains data on suspect and concomitant drugs, drug dosage, duration and indications for use, patient demographics, outcomes, name and country of the reporting source. Not all fields are mandatory for submitting an $\mathrm{AE}$ report, and many reports are incomplete. FAERS data are publicly released on a quarterly basis as files that can be processed with methods for orthogonal database search.

The study was conceived as an observational, retrospective pharmacovigilance analysis, which combined descriptive case series with DA. Considering the expected rarity of FG, individual inspection of reports is useful to characterise patient-related and drug-related factors for causality, whereas DA is a recognised approach for rapid and timely detection of rare AEs and to preliminarily assess the magnitude of reporting as compared with other drugs with similar therapeutic indications. ${ }^{16}$

\section{Retrieval and characterisation of $\mathrm{FG}$ reports}

To identify all possible reports for SGLT2i listing FG as an AE, we searched the FAERS using different methods. The search terms for data retrieval and disproportionality analysis are detailed in online supplementary material. 'Necrotizing fasciitis' was the preferred term in Medical Dictionary for Regulatory Activities (MedDRA) V.20.1 for 'Fournier's gangrene', which has entered MedDRA 21.1 terms since September 2018. This implies that an automated search on databases using MedDRA terms up to $2018 \mathrm{q} 3$ could identify NF as FG only when the perineal site of NF was specified. We first searched the FAERS up to 2018q3 on the FDAble platform for AE reports listing SGLT2i among suspect or concomitant drugs, and the following MedDRA terms: 'Fournier's gangrene' or 'scrotal gangrene' or 'scrotal abscess' or 'perineal abscess' or 'perineal cellulitis' or 'perineal infection' or 'necrotising fasciitis' or 'necrotising fasciitis streptococcal' or 'necrotising fasciitis staphylococcal' or 'gangrenous balanitis' or 'vulval cellulitis' or 'cellulitis of male external genital organ'.

In parallel, we searched the public FAERS dashboard for all available AEs including 'Fournier's gangrene' as a preferred term up to $2018 \mathrm{q} 4$. This allowed to definitely classify as FG cases initially retrieved as suspected FG based on MedDRA terms up to 2018q3. To further refine the search and increase specificity, on 4 March 2019 we asked the FDA for a freedom of information (FOI) in order to obtain structured and unstructured details on the retrieved events that could not be definitely classified as SGLT2i-associated FG. The FOI was released on 7 May 2019, when the FDA published details on 55 events of FG reported in patients taking SGLT2i up to 31 January 2019. ${ }^{15}$ Using narratives of single AE report, we could further classify as definite FG the remaining suspected cases or discharge them as non-genital $\mathrm{AE}$ or as severe AEs of the genital area, other than FG. Narratives criteria to define FG were the verbatim for 'Fournier's gangrene' or the description of a necrotising infection of the perineum. Need for surgery was not a criterion to define FG because, like many other fields of many AE reports, diagnostic and therapeutic measures undertaken to treat the $\mathrm{AE}$ are often omitted.

The final list of SGLT2i-associated FG did not intentionally include cases reported in $2018 \mathrm{q} 4$ or later to limit the expected stimulated reporting after the FDA warning (the so-called notoriety bias). ${ }^{17}$

\section{Disproportionality analysis}

To evaluate if and to what extent FG has been reported for SGLT2i more often than for other drugs, we performed a DA, a consolidated approach in pharmacovigilance to identify potential drug-event associations, especially in the diabetes area. ${ }^{18}$ Although pharmacovigilance evaluation cannot inform on causality, if a drug $\mathrm{X}$ causes the event $\mathrm{Y}, \mathrm{Y}$ will be found more frequently in reports listing $\mathrm{X}$ than in reports listing non-X drugs as suspect or concomitant, thus generating disproportionality. Vice 
versa, if no link exists between $\mathrm{X}$ and $\mathrm{Y}$, the event $\mathrm{Y}$ will have the same frequency in reports listing as in those not listing $\mathrm{X}$ as a suspect or concomitant drug.

We used the public web-based resource AERSmine, which allows customised analyses by filtering the $\mathrm{AE}$ search for drugs (suspected or concomitant) and their indications (MedDRA terms), AEs (MedDRA terms), outcomes, demographics and periods. ${ }^{19}$ The same definition of FG based on MedDRA terms was used as for the search in FDAble. Several search strategies were planned and performed, with different sensitivity and specificity: (a) FG among reports for SGLT2i versus reports for any other drug; (b) as in (a) but retaining only reports for drugs used for the treatment of diabetes (for definition of the indication filter, see the online supplementary material); (c) FG among reports for SGLT2i versus reports for other GLMs (ATC class plus insulin, for details see online supplementary material). For each search, a subanalysis was performed restricting to the time window from 2013q1 (when SGLT2i entered the market) to 2018q3 (at the end of which the FDA warning was published). The search strategy (c) from 2013q1 to 2018q3 was further refined using a restrictive definition of FG by MedDRA terms, obtained from the match between FDAble, the public FDA dashboard and the FOI ("necrotising fasciitis" OR "necrotising fasciitis streptococcal" OR "necrotising fasciitis staphylococcal" OR "scrotal abscess" OR "scrotal gangrene"). In addition, AEs for amputations ("amputation" OR "limb amputation" OR "leg amputation" OR "foot amputation" OR "finger amputation" OR "toe amputation" OR "arm amputation" OR "hand amputation") were excluded because AEs listing NF of unknown site in association with an amputation were considered $\mathrm{NF}$ of the limbs and therefore unrelated to FG. For each search, we retrieved the number of FG cases over total reports and calculated frequencies of FG/1000 reports with $95 \%$ CI. Disproportionality was calculated using the proportional reporting ratio (PRR) and its 95\% CI.

\section{RESULTS}

\section{Analysis of retrieved cases}

Online supplementary figure 1A shows the flowchart used to identify definite FG cases. Using a wide definition of FG based on MedDRA terms, we initially retrieved 84 cases of suspected FG reported in association with SGLT2i from 2004q1 to 2018q3. After removing two duplicates and excluding nine cases that were deemed unrelated to the genital area based on a review of the $\mathrm{AE}$ terms, 73 suspect FG cases were further evaluated against the public dashboard and narratives released by the FDA. Forty-seven of the 73 reports were confirmed as FG: 40 by the preferred term specified in the public dashboard (which is not limited to MedDRA terms) and seven that could be definitely classified as FG based on a review of the AE narratives. Aggregated characteristics of these cases are shown in table 1, whereas individual reports are shown in online supplementary table S1. Patients were
Table 1 Aggregate characteristics of reports for definite Fournier's gangrene $(F G)$ and severe genital adverse events (AEs), with SGLT2i as suspect or concomitant drugs

\begin{tabular}{|c|c|c|}
\hline & Definite FG & $\begin{array}{l}\text { Severe genital } \\
\text { AEs }\end{array}$ \\
\hline Total cases & 47 & 17 \\
\hline Reporter country (US/non-US) & $29 / 18$ & 06/11/19 \\
\hline Age, years (mean/range) ${ }^{*}$ & $58.9 / 38-78$ & $50.7 / 35-70$ \\
\hline Sex (M/F/)†† & $31 / 11$ & $12 / 03 / 19$ \\
\hline \multicolumn{3}{|l|}{ Type of SGLT2i } \\
\hline Empagliflozin & 14 & 3 \\
\hline Dapagliflozin & 17 & 7 \\
\hline Canagliflozin & 16 & $7 \dagger$ \\
\hline Primary/secondary suspect & $47 \ddagger$ & $16 \dagger$ \\
\hline \multicolumn{3}{|l|}{ Diabetes type } \\
\hline Type 1 diabetes & 0 & 0 \\
\hline Type 2 diabetes & 25 & 10 \\
\hline Not specified & 7 & 1 \\
\hline Not reported & 15 & 6 \\
\hline \multicolumn{3}{|l|}{ Concomitant medications $\ddagger \ddagger$} \\
\hline Glucose-lowering agents & 21 & 7 \\
\hline Lipid-lowering agents & 7 & 3 \\
\hline $\begin{array}{l}\text { Cardiovascular agents } \\
\text { (including ASA) }\end{array}$ & 6 & 3 \\
\hline $\begin{array}{l}\text { Systemic } \\
\text { immunosuppressant drugs }\end{array}$ & $2 \S \S$ & 1 \\
\hline Systemic anti-infectives & 2 & 0 \\
\hline Levothyroxine & 2 & 0 \\
\hline Antiasthma & 191 & 0 \\
\hline Others & 11 & 3 \\
\hline None/not reported & 26 & 11 \\
\hline $\begin{array}{l}\text { Mean time to onset, days } \\
\text { (range)§ }\end{array}$ & $434(0-2508)$ & $291(0-965)$ \\
\hline \multicolumn{3}{|l|}{ Outcomes } \\
\hline Hospitalisation & 36 & 13 \\
\hline Life-threatening & 19 & 0 \\
\hline Disability & 5 & 2 \\
\hline Requiring intervention & 5 & 0 \\
\hline Death $^{\star \star}$ & 2 & 0 \\
\hline Other & 14 & 6 \\
\hline
\end{tabular}

${ }^{*}$ Not reported in 20 cases.

†In one case canagliflozin was reported as concomitant.

$\ddagger$ In one case dapagliflozin was reported as secondary suspect. §Calculated for 27 reports with available data on 'start date' and 'event date'. In case the specific day of start/event dates was not recorded, day 1 was imputed. Reports with incomplete data (both year and month of reporting) were excluded.

ПA report may be coded with multiple outcomes.

${ }^{* *}$ One death was reported in the case narrative.

$\dagger †$ Not reported in seven cases.

$\ddagger \ddagger A$ report may contain multiple medications. If more than one drug of a given therapeutic class was reported (eg, glucose-lowering agents), the case counted only once.

$\S \S$ In one case, secukinumab was reported as primary suspect drug. ๆףIncluding a short-acting $\beta 2$-adrenoreceptor agonist and a combination of inhaled corticosteroid and long-acting $\beta 2$ adrenoreceptor agonist.

ASA, acetylsalicylic acid. 
on average 58.9 years old; 31 were men and 11 women ( $\mathrm{n}=5$ not reported).

Among the cases that were not classified as FG, $n=17$ were severe inflammatory/infectious AEs involving the genital area: cellulitis of male $(n=7)$ or female $(n=1)$ external genital organ(s), scrotal or perineal abscess $(n=5)$, other perineal infections $(n=2)$ and NF of anatomical areas close to the perineum (pubis and pelvis, $n=2$ ). Aggregated characteristics of these cases are compared with those of FG cases (table 1). These patients were significantly younger than those with definite FG (50.7 vs 58.9 years; $\mathrm{p}=0.04)$, whereas gender distribution was similar (12 men, 3 women, 2 unknown).

The remaining nine cases were classified as NF of nongenital areas $(n=4)$, NF of unknown site $(n=4)$ or duplicate $(\mathrm{n}=1)$.

With the exception of ertugliflozin, all SGLT2i currently approved in the USA were represented and reported as primary/secondary suspect; in one case, the SGLT2i (canagliflozin) was reported as concomitant. The majority of cases were serious (ie, death, hospitalisation-initial or prolonged-life-threatening or disability were recorded), especially for definite FG ( $81 \%$ vs $75 \%)$, with hospitalisation recorded in $77 \%$ of reports. Only two deaths were recorded. Overall, information about concomitant medications and indications were scant. No concomitant drugs were recorded in $55 \%$ of definite FG cases. Of note, two reports listed SGLT2i concomitantly with drugs for the treatment of severe psoriasis (secukinumab and/or topical betamethasone), and another definite FG case listed prednisone.

\section{Disproportionality analysis}

Among $11525276 \mathrm{AE}$ reports filed to the FDA between 2004q1 and 2018q3, 45927 listed SGLT2i as suspect or concomitant drugs, 91 of which satisfied the wide definition of FG, equal to a reporting frequency of 2.0/1000 (95\% CI 1.6 to 2.4). Among the 11479349 reports filed for non-SGLT2i drugs, there were 2862 putative cases of $\mathrm{FG}$, equal to a frequency of $0.2 / 1000(95 \%$ CI 0.2 to 0.3 ). The resulting PRR was 7.95 (95\% CI 6.45 to 9.79). Restricting this analysis to the period when SGLT2i were available in the market (2013q1-2018q3) but still excluding the quarters after the FDA warning, results did not change substantially because the rate of FG reports filed for non-SGLT2i drugs remained stable (table 2). The flowchart in online supplementary material 1 shows refinement of the DA.

Since diabetes is a risk factor for $\mathrm{FG},{ }^{20}$ we wished to verify whether the disproportional association between FG reports and SGLT2i reflected diabetes-associated FG risk rather than SGLT2i-associated FG risk. First, we found that FG was reported disproportionally more for drugs having the diabetes indication, but the difference was small (PRR 1.48; 95\% CI 1.28 to 1.71). In fact, when we restricted the search to reports for drugs with the diabetes indication, the rates of FG associated with SGLT2i and non-SGLT2i drugs did not change substantially and the
PRR was 8.29 from the period 2004q1-2018q3 and 8.44 for the period 2013q1-2018q3.

We calculated that, in the FAERS, only $52 \%$ of the AE reports filed for GLM contain the diabetes indication. Although SGLT2i may be used off-label outside the diabetes indication (eg, for the treatment of obesity), this lack of diabetes indication is likely due to incomplete reporting. Thus, we compared SGLT2i with other GLMs (drugs of the ATC10 class and insulin): the number of retrieved events in the non-SGLT2i GLM control group was indeed higher than in reports with the diabetes indication, but reporting rates did not change substantially and the PRR was 6.99 for $2004 q 1-2018 q 3$ and 8.23 for 2013q1-2018q3.

Finally, we applied a more restrictive definition of FG, which included only MedDRA terms that were more likely to correspond to FG as determined by matching the various sources of information as described above and excluded terms related to amputations. With the refined definition, sensitivity against FG defined by preferred term and narratives remained high (from $100 \%$ to $95.7 \%$ ) and specificity increased from $0 \%$ to $46.4 \%$. Even with this definition, the PRR of FG for SGLT2i versus other GLMs was 5.17. When further restricted to 2013q1-2018q3 and limited to AE reporting the diabetes indication (figure 1), the number of retrieved cases was 46 for SGLT2i and 74 for other GLMs, with corresponding rates of 1.5/1000 versus $0.2 / 1000$, equal to a PRR of FG for SGLT2i of 6.42 (95\% CI 4.44 to 9.27 ).

Figure 2 shows that the rates of FG reports (using the strict definition) over time became significantly higher for SGLT2i versus other GLMs since 2015, increased for SGLT2i until 2018q3 and then showed a fourfold sudden increase in 2018q4, after the FDA warning. This likely reflects stimulated reporting and justifies the exclusion of such period from DA.

To rule out that similar results could be obtained for other specific classes of GLMs, we performed a control analysis on DPP-4 inhibitors. The PRR of FG for DPP-4i was 1.36 (95\% CI 0.96 to 1.91$)$ versus any other drug and $0.52(95 \%$ CI 0.30 to 0.91$)$ versus other GLMs, suggesting that the association with FG was specific for SGLT2i.

Since we detected that three patients with SGLT2iassociated FG or severe genital AEs received systemic immunosuppressant drugs, including agents to treat severe psoriasis such as secukinumab, we performed a DA for FG (strict definition) related to psoriasis. This analysis was extended to $2018 \mathrm{q} 4$ because it is unrelated to the warning for SGLT2i. We found a mild disproportionality of FG cases in reports for drugs with the psoriasis indication versus those with other indications (PRR 1.59; 95\% CI 1.29 to 1.97 ). Furthermore, the rate of FG was 2.55 times as high (95\% CI 1.36 to 4.78 ) as in reports for GLMs when a concomitant drug indicated for psoriasis was present. In reports concomitantly listing drugs with indication for diabetes and drugs with indication for psoriasis, the use of interleukin inhibitors was associated with a PRR for FG of 13.7 (95\% CI 2.7 to 70.5). Finally, 
Table 2 Results of the disproportionality analysis

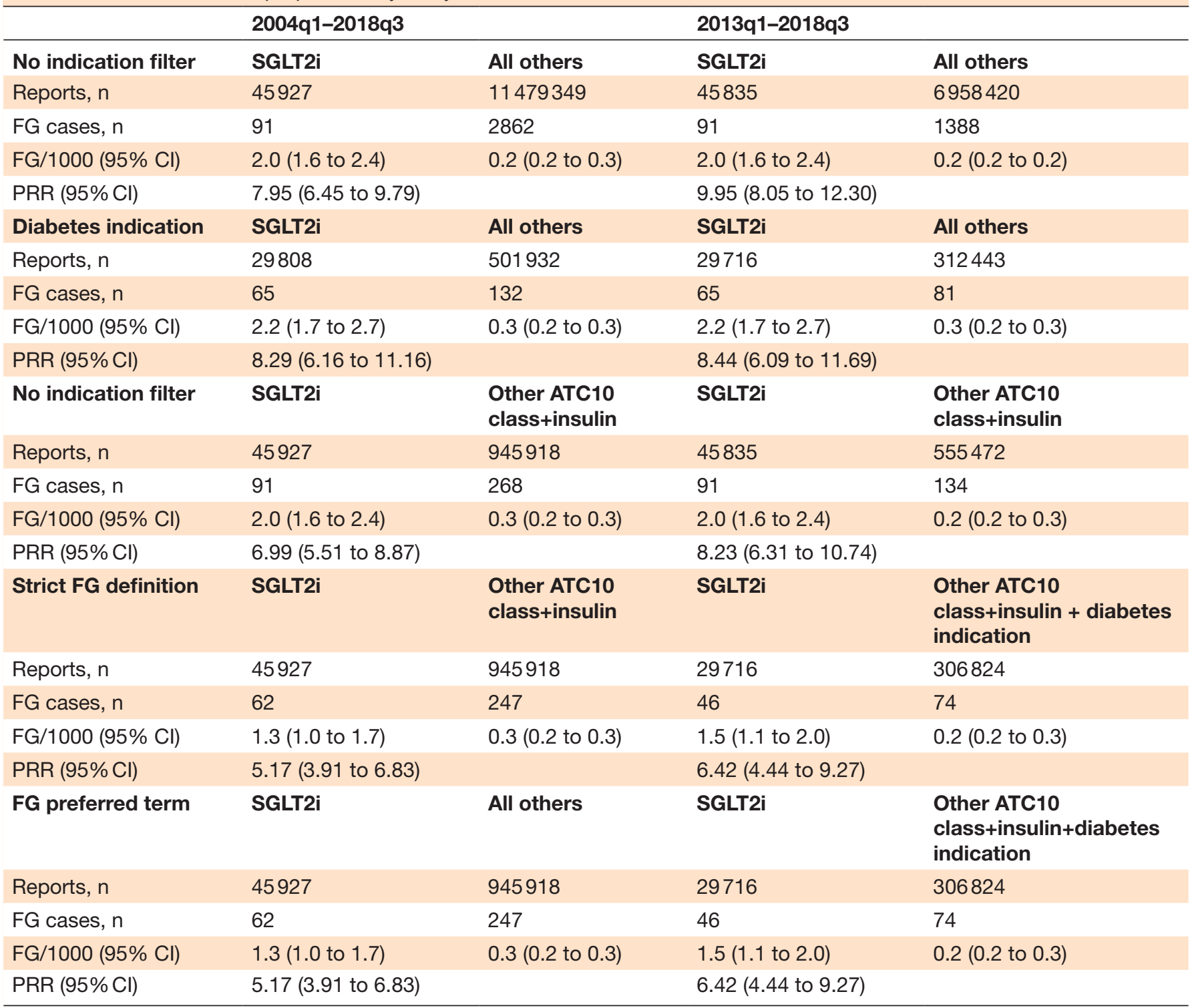

For each analysis, the table reports the number of total adverse event (AE) reports, the number of reports listing Fournier's gangrene (FG) as an $\mathrm{AE}$, the rate with $95 \% \mathrm{Cl}$ of $\mathrm{FG}$ and the proportional reporting ratio (PRR) with $95 \% \mathrm{Cl}$.

three cases of FG were identified in reports concomitantly listing SGLT2i and interleukin inhibitors (such as secukinumab), with a frequency of $16.0 / 1000$, yielding a PRR of 6.79 (95\% CI 2.12 to 21.17) versus the rate of FG associated with SGLT2i without concomitant interleukin inhibitors.

\section{CONCLUSIONS}

For their benefits on cardiorenal outcomes and according to international recommendations, SGLT2i should be prioritised over other GLMs in the treatment of T2D, especially in patients with heart failure and chronic kidney disease. ${ }^{21}$ Since it is expected that the number of SGLT2i users will increase substantially in the future, paying attention to severe AEs is very important even if they are extremely rare.
In this pharmacovigilance analysis, we show that, since 2015, FG has been reported significantly more often by patients taking SGLT2i than patients taking other medications. Our analysis yielded consistent and robust estimates of disproportionality even after accounting for reporting period, higher rates of FG associated with diabetes, and after refining the definition of FG.

Remarkably, it appears that the rate of FG reports for SGLT2i, but not for other GLMs, was already increasing before the 2018 FDA warning. This trend should not be interpreted as a direct consequence of the increased use of SGLT2i worldwide, but may reflect that SGLT2i were being used in progressively less selected populations, with FG-predisposing factors such as impaired immune system. 


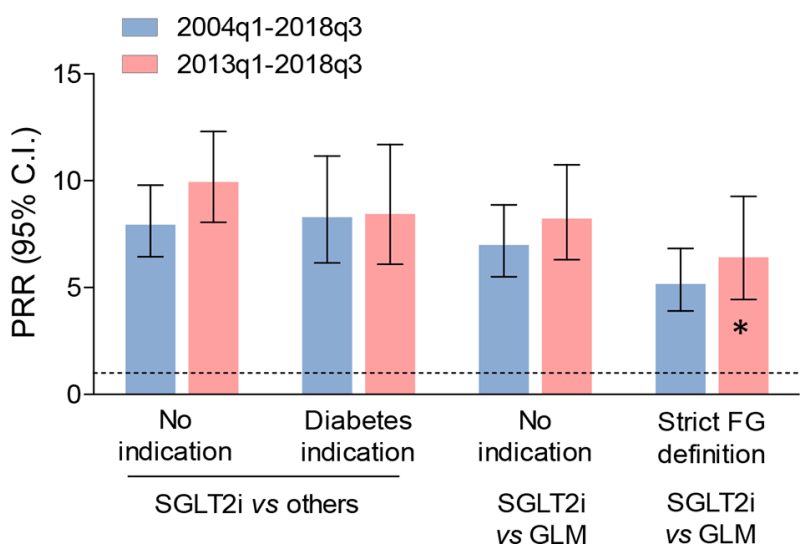

Figure 1 Results of the disproportionality analysis. Proportional reporting ratios (PRRs) are shown with bars indicating $95 \% \mathrm{Cl}$. Different analyses are shown as reported in detail in table 2and described in the text. For each analysis, the PRR is shown for the entire FAERS but before stimulated reporting could take place (2004q1-2018q3) or starting from when SGLT2i entered the market (2013q12018q3). FG, Fournier's gangrene; GLM, glucose-lowering medication (defined as ATC10 class and insulins); SGLT2i, sodium glucose contransporter-2 inhibitors. *This analysis was further filtered for the diabetes indication.

FG is an extremely dangerous NF of the perineum typically involving external genital organs. Its aggressive course can lead to sepsis and death if not promptly treated with antibiotics and surgery. Patients surviving from FG often have disfiguring or permanently disabling outcomes. ${ }^{20}$ Up to $2018 q 3$, we found 47 cases of FG in patients taking SGLT2i and detected a sharp fourfold increase in the reporting frequency during 2018q4 (with 51 additional cases), which can be probably accounted

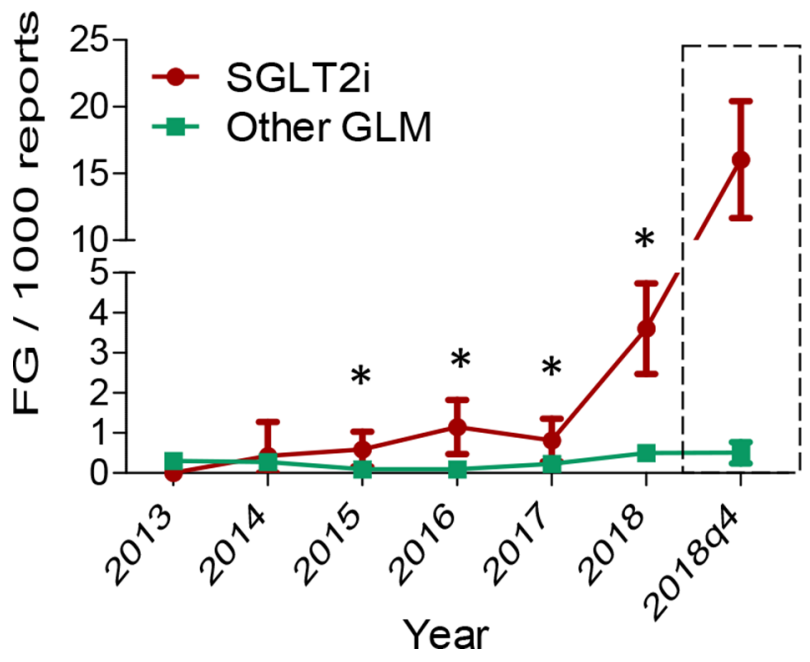

Figure 2 Time trend in rates of Fournier's gangrene (FG) reported to the FDA. The rates of FG reports per 1000 reports are shown for sodium glucose cotransporter-2 inhibitors (SGLT2i) and for other glucose-lowering medications (GLMs) over time. The dashed box illustrates the surge in FG cases among reports filed for SGLT2i during $2018 q 4$, that is, after the warning issued by the U.S. Food and Drug Administration, indicating stimulated reporting. for by the FDA warning issued at the end of August 2018. Although true FG may not resolve spontaneously without surgery, our definition of FG did not require the reports to specify that patients underwent surgery because underreporting of diagnostic and therapeutic procedures for AEs is common. In contrast, a recent publication by the FDA reported 55 cases of SGLT2i-associated FG up to the end of January $2019 .{ }^{15}$ This report defined FG as a necrotising infection of the perineum that required surgery, but allowed extension beyond the perineum (eg, to the buttocks) and not necessarily reaching the fascial compartment, a criterion needed to define NF. Thus, the authors found a relatively small number of SGLT2iassociated FG cases despite they used a less rigorous definition than ours and captured 4 months of stimulated reporting because they excluded 41 cases for not having documented surgery. ${ }^{15}$ An underestimation of the number of FG events was also evident among reports for other GLMs $(n=19)$, as compared with our analysis $(\mathrm{n}=247)$.

While searching for FG, we found reports suggestive of other severe infections of the genital area occurred in SGLT2i recipients. Even though events of genital cellulitis, abscess and gangrene could not be referred to FG, they should not be overlooked and require as close monitoring as FG does. It is important to note that these AEs, somewhat less severe than FG, occurred in patients who were on average 10 years younger, in agreement with the notion that typical FG occurs more often in aged individuals. ${ }^{20} 22$

The analysis of individual cases identified no obvious hint of common precipitating factors that may highlight SGLT2i users at higher risk for FG, except that we found three patients taking immunosuppressive drugs, including drugs for the systemic treatment of psoriasis. Although this number is small, the finding might be more than a coincidence. Indeed, the combination of steroids and/or interleukin inhibitors (eg, to treat severe psoriasis) with SGLT2i can make the patients prone to infections. ${ }^{23}$ In determining the rates of FG reports, an interplay was noted among diabetes, psoriasis and the respective drugs. Psoriasis can affect genital organs ${ }^{24}$ and scratching can lead to skin lesions that allow bacteria to reach deeper planes. Psoriasis is relatively common among patients with $\mathrm{T}_{2} \mathrm{D}^{25}$ who would benefit from SGLT2i therapy. We therefore suggest that attention should be paid to patients with genital psoriasis receiving systemic immunosuppressants. However, this signal is based on a very small number of cases and needs to be confirmed. If any, it could identify a small minority of diabetic patients at risk for SGLT2i-associated FG.

Lack of the diabetes indication in individual reports listed in online supplementary table S1 should not be interpreted as if the patient was not diabetic because incomplete/missing reporting of drug indications is very common. Indeed, four cases listed SGLT2i along with other GLMs among suspect or concomitant, even when the diabetes indication was not reported. 
Several caveats apply to the interpretation of pharmacovigilance analyses. First, a causal relationship cannot be inferred and indication of SGLT2i as suspect for FG was at discretion of the individuals who filed the reports. It is therefore impossible to judge whether SGLT2i caused FG or simply were the ongoing therapy in patients who developed FG for other reasons. Online supplementary table S2 illustrates causality assessment with BradfordHill criteria. Rates of SGLT2i-associated FG within the pharmacovigilance database do not inform on the true risk in clinical practice, which can only be derived from prospective studies where the population at risk is clearly defined. Nonetheless, comparisons can be made within the pharmacovigilance setting: the disproportional association of SGLT2i with FG was quantitatively similar to that calculated for ketoacidosis ${ }^{8}$ and stronger than that of amputations. ${ }^{9}$

Epidemiological studies report an annual incidence of FG of 1.6 to 3.3/100 000 patients, with a $3 \times$ higher risk in diabetics. ${ }^{22}$ In the DECLARE study, six cases were reported among 17160 diabetic patients randomised and treated for 4.2 years, ${ }^{3}$ equal to an annual rate of $8.3 / 100000$, perfectly in line with what is expected in diabetic patients. The nominally lower rate of FG in the dapagliflozin group $(n=1)$ than in the placebo group $(n=5)$ is reassuring. ${ }^{3}$ This may in fact reflect that aged patients with FG predisposing conditions were excluded from the trial or intercepted early. If the disproportionality observed in the FAERS applied to clinical practice, it could be calculated that 1 every 1200 to 1800 patients treated with SGLT2i may experience FG each year.

Another limitation is that in the FAERS, indications for drug use may drive differential reporting of AEs, leading to indication-specific rather than drug-specific signatures. Yet, the disproportional reporting of FG for SGLT2i was consistent with or without the diabetes indication and in the comparison with both any other drug or medications for the treatment of diabetes. In addition, the association of diabetes with FG was statistically significant but much weaker than the association of SGLT2i with FG. It should also be mentioned that part of the AEs have been filed by non-health care professionals, thereby limiting their reliability. Moreover, the analysis of the interaction between drugs (SGLT2i and interleukin inhibitors) and drug indications (diabetes and psoriasis) is subjected to the bias of dilution by other drug-related AEs. Finally, confounding by indication cannot be excluded considering that SGLT2i may be preferred among GLMs for patients with important comorbidities, such as CVD.

A few clinical messages can be derived from this study. First, based on the solid DA and on the well-known risk of genital infections associated with SGLT2i, physicians should be aware that SGLT2i could predispose to FG and other severe genital AEs. Although FG remains rare, the predictable increase in the number of patients who will receive SGLT2i in the future implies that close monitoring and constant epidemiological surveillance are needed. In addition to paying attention to elderly patients, we suggest that patients with genital psoriasis, especially if on immunosuppressant therapy, and other patients prone to invasive infections should be carefully informed at the time of SGLT2i prescription and more closely monitored. Finally, we wish to remind that detailed AE reporting can provide valuable opportunities to identify safety signals, quantify the problem, establish risk factors and ultimately protect patients' safety.

Contributors GPF: study design, data collection, analysis and interpretation, manuscript writing. MS: data collection and analysis, manuscript revision. FDP: data analysis and interpretation, manuscript revision. AA: study design, data interpretation, manuscript revision. ER: study design, data collection, analysis and interpretation, manuscript writing. All authors provided substantial contributions to conception and design, acquisition of data or analysis and interpretation of data, drafting the article or revising it critically for important intellectual content. All authors approved the final version to be published.

Funding The study was supported by institutional grants from the University of Padova and received no specific funding. The corresponding author had full access to all of the data and the final responsibility to submit for publication.

Competing interests GPF reports grants, personal fees and non-financial support from AstraZeneca, personal fees and non-financial support from BoehringerIngelheim, personal fees from Mundipharma during the conduct of the study; grants, personal fees and non-financial support from Eli Lilly, personal fees and non-financial support from NovoNordisk, personal fees and non-financial support from Sanofi, non-financial support from Genzyme, personal fees and non-financial support from Abbott, personal fees and non-financial support from Novartis, personal fees from Merck Sharp \& Dohme, outside the submitted work. AA reports grants, personal fees and non-financial support from AstraZeneca, personal fees from Boehringer-Ingelheim, personal fees from Janssen, during the conduct of the study; personal fees from Merck Sharp \& Dome, personal fees and non-financial support from Novartis, personal fees from Sanofi, grants and personal fees from Mediolanum, personal fees from NovoNordisk, personal fees from Lilly, personal fees and non-financial support from Servier, personal fees from Takeda, outside the submitted work

Patient consent for publication Not required.

Provenance and peer review Not commissioned; externally peer reviewed.

Data availability statement All data relevant to the study are included in the article or uploaded as online supplementary information.

Open access This is an open access article distributed in accordance with the Creative Commons Attribution Non Commercial (CC BY-NC 4.0) license, which permits others to distribute, remix, adapt, build upon this work non-commercially, and license their derivative works on different terms, provided the original work is properly cited, appropriate credit is given, any changes made indicated, and the use is non-commercial. See: http://creativecommons.org/licenses/by-nc/4.0/.

ORCID iDs

Gian Paolo Fadini http://orcid.org/0000-0002-6510-2097

Emanuel Raschi http://orcid.org/0000-0003-0487-7996

\section{REFERENCES}

1. Vasilakou D, Karagiannis T, Athanasiadou E, et al. Sodium-glucose cotransporter 2 inhibitors for type 2 diabetes: a systematic review and meta-analysis. Ann Intern Med 2013;159:262-74.

2. Zelniker TA, Wiviott SD, Raz I, et al. SGLT2 inhibitors for primary and secondary prevention of cardiovascular and renal outcomes in type 2 diabetes: a systematic review and meta-analysis of cardiovascular outcome trials. Lancet 2019;393:31-9.

3. Wiviott SD, Raz I, Bonaca MP, et al. Dapagliflozin and cardiovascular outcomes in type 2 diabetes. N Engl J Med 2019;380:347-57.

4. Perkovic V, Jardine MJ, Neal B, et al. Canagliflozin and renal outcomes in type 2 diabetes and nephropathy. N Engl J Med 2019;380:2295-306.

5. Li D, Wang T, Shen S, et al. Urinary tract and genital infections in patients with type 2 diabetes treated with sodium-glucose cotransporter 2 inhibitors: a meta-analysis of randomized controlled trials. Diabetes Obes Metab 2017;19:348-55. 
6. Zaccardi F, Webb DR, Htike ZZ, et al. Efficacy and safety of sodiumglucose co-transporter-2 inhibitors in type 2 diabetes mellitus: systematic review and network meta-analysis. Diabetes Obes Metab 2016;18:783-94.

7. Ruanpeng D, Ungprasert P, Sangtian J, et al. Sodium-glucose cotransporter 2 (SGLT2) inhibitors and fracture risk in patients with type 2 diabetes mellitus: a meta-analysis. Diabetes Metab Res Rev 2017;33. doi:10.1002/dmrr.2903

8. Fadini GP, Bonora BM, Avogaro A. SGLT2 inhibitors and diabetic ketoacidosis: data from the FDA adverse event reporting system. Diabetologia 2017;60:1385-9.

9. Fadini GP, Avogaro A. SGLT2 inhibitors and amputations in the US FDA adverse event reporting system. Lancet Diabetes Endocrinol 2017;5:680-1.

10. Bonora BM, Avogaro A, Fadini GP. Sodium-glucose co-transporter-2 inhibitors and diabetic ketoacidosis: an updated review of the literature. Diabetes Obes Metab 2018;20:25-33.

11. Yuan Z, DeFalco FJ, Ryan PB, et al. Risk of lower extremity amputations in people with type 2 diabetes mellitus treated with sodium-glucose co-transporter-2 inhibitors in the USA: a retrospective cohort study. Diabetes Obes Metab 2018;20:582-9.

12. Onder CE, Gursoy K, Kuskonmaz SM, et al. Fournier's gangrene in a patient on dapagliflozin treatment for type 2 diabetes. $J$ Diabetes 2019;11:348-50.

13. Kumar S, Costello AJ, Colman PG. Fournier's gangrene in a man on empagliflozin for treatment of type 2 diabetes. Diabet Med 2017;34:1646-8.

14. U.S. Food and Drug Administration. FDA warns about rare occurrences of a serious infection of the genital area with SGLT2 inhibitors for diabetes, 2018. Available: https://www.fda.gov/Drugs/ DrugSafety/ucm617360.htm [Accessed May 2019].

15. Bersoff-Matcha SJ, Chamberlain C, Cao C, et al. Fournier gangrene associated with sodium-glucose cotransporter-2 inhibitors: a review of spontaneous postmarketing cases. Ann Intern Med 2019;170:764-9.
16. Dias P, Penedones A, Alves C, et al. The role of disproportionality analysis of pharmacovigilance databases in safety regulatory actions: a systematic review. Curr Drug Saf 2015;10:234-50.

17. Raschi E, Piccinni C, Poluzzi E, et al. The association of pancreatitis with antidiabetic drug use: gaining insight through the FDA pharmacovigilance database. Acta Diabetol 2013;50:569-77.

18. Raschi E, Poluzzi E, Salvo F, et al. Pharmacovigilance of sodiumglucose co-transporter-2 inhibitors: what a clinician should know on disproportionality analysis of spontaneous reporting systems. Nutr Metab Cardiovasc Dis 2018;28:533-42.

19. Sarangdhar M, Tabar S, Schmidt C, et al. Data mining differential clinical outcomes associated with drug regimens using adverse event reporting data. Nat Biotechnol 2016;34:697-700.

20. Eke N. Fournier's gangrene: a review of 1726 cases. Br J Surg 2000;87:718-28.

21. Davies MJ, D'Alessio DA, Fradkin J, et al. Management of hyperglycemia in type 2 diabetes, 2018. A consensus report by the American Diabetes Association (ADA) and the European Association for the Study of Diabetes (EASD). Diabetes Care 2018;41:2669-701.

22. Sorensen MD, Krieger JN, Rivara FP, et al. Fournier's gangrene: population based epidemiology and outcomes. J Urol 2009;181:2120-6.

23. Vanhove T, Remijsen Q, Kuypers D, et al. Drug-drug interactions between immunosuppressants and antidiabetic drugs in the treatment of post-transplant diabetes mellitus. Transplant Rev 2017;31:69-77.

24. Meeuwis KAP, de Hullu JA, Massuger LFAG, et al. Genital psoriasis: a systematic literature review on this hidden skin disease. Acta Derm Venereol 2011;91:5-11.

25. Neimann AL, Shin DB, Wang X, et al. Prevalence of cardiovascular risk factors in patients with psoriasis. J Am Acad Dermatol 2006;55:829-35. 